\title{
VISUALIZATION OF VIRTUAL PLANT BASED ON 3D MODEL CONVERTER
}

\author{
Qian Wang ${ }^{1}$, Ying Zhang ${ }^{1, *}$, Ji Liu ${ }^{1}$ \\ ${ }^{1}$ Computer College of Chongqing University, Chongqing, P. R. China 400030 \\ * Corresponding author, Address: 400030, Computer College of Chongqing University, \\ Chongqing, P. R. China, Tel: +86-23-65102506,Email: linzhibinjelly@163.com
}

\begin{abstract}
We present a method based on a 3D model converter to simulate the development of virtual plant. The converter is mainly used to import the plant organs which have fine details into the virtual plant development system, then communicating with the L-system to implement the simulation of the development of plants controlled by the physiology of plant. It improves the virtual effect that carried out by the former systems which only take into account the geometric model.
\end{abstract}

Keywords: modeling of virtual plant organs, L-system, 3D model converter, visualization of virtual plant

\section{INTRODUCTION}

Plant development is a dynamic process in which the topology and geometry change over time in a seemingly complex manner. We can simulate the developing process in the three-dimensional space on computer to reflect the morphological structure changing of the realistic scene, including the individual plant and the community (Ma Xinming et al.,2003).

Models of plant development can be implemented using a variety of methods. They contain the Particle Systems (Reeves, 1985),A-System (Aono et al.,1984), the Realistic method for the Procedural Generation of Trees (Weber et al.,1995) and the AMAP (De Reffye et al., 1988;Jaeger et al.,1992) model etc, but they are all modeling plant organs through the geometrical parameters, not considering the fine detail on them, which leads to lacking of

\footnotetext{
Please use the following format when citing this chapter:

Wang, Q., Zhang, Y. and Liu, J., 2009, in IFIP International Federation for Information Processing, Volume 294, Computer and Computing Technologies in Agriculture II, Volume 2, eds. D. Li, Z. Chunjiang, (Boston: Springer), pp. 1467-1476.
} 
the visual effect. An improved model Greenlab (De Reffye et al.,2003) based on the AMAP was proposed by the researchers, it's a function-structural model that uses the mathematical expression to represent the developing process which excels the AMAP in simulating the complicate morphological structure. Also it has limitation, it can only create the organs that have similar shapes and the surface detail was not incarnated. Moreover, there are plenty of modeling softwares that support the texture mapping that can express the detail on the surface which can enhance the virtual effect, but it can not implement the plant developing process which has relation with time, so they can not be used to simulate the developing process of plant directly.

To solve the above problems, we present a new method which models the plant elementary organs (like stem, petal, calyx, leaf et al.) by the outside modeling software and choose the L-system(Lindenmayer, 1968; Prusinkiew et al.,1990; Karwowski et al.,2003) to simulate the plant development. According to this formalism, a plant is viewed as a developing assembly of individual units, or modules. These modules are characterized by parameters such as length, width, and age, as well as parameters characterizing shape (Mundermann et al., 2005). Here we use a three-dimension model converter to import the plant organs designed by the outside modeling software to the L-system to control the plant growing. The experiment result shows this methodology can effectively solve the problem of lacking of the detail on the plant surface in real time and combine the physiology of plant seamlessly.

\section{IMPLEMENTATION}

\subsection{Visual approach}

A plant can be considered as a series of organs, each consists of many elementary components that array regularly in the three-dimensional space. Those are stems, leaves, flowers et al.. Now we will introduce our method to model the plant organs by the outside modeling software. All models are created in relative size not the actual size. All of the organs' centre must be in the origin of the world space. And all organs' texture comes from the photos taken by us. This will enhance the visual effect.

1) Leaves

The obvious characteristic of leaf is its geometrical shape and surface texture. So we design the leaf according to the photos taken from the plant of various view directions. First, we use a two dimension plane to simulate the leaf, then we extend it in the third dimension. (see Fig.1(a)).

As for the organs' texture, we will use the two-part texture mapping method (Bier et al.,1986) to transfer the texture space to the world space, we 
will use the plane as the intermediary surface, that is, first map the texture onto the plane, then use the texture image from the plane to map onto the leaf's surface.

\section{2) Flowers}

One of the most important parts of plant is the flower. It comprises by many components, namely, the petal, the pistil, the stamen, and the calyx after our simplifying. Here we illustrate our means to determine these organs.

Petal's shape is similar to the leaf, so we use the same way of the leaf's creating. And both of the pistil and the stamen (see Fig.1(b)) are gotten by metamorphosing the sphere. As to the calyx, its body can be constructed by two steps: an axis from the flower branch to the chaplet and the shape with different sizes circling the axis. So we can determine it by appointing the shape of the axis and the different radius of the shape infixed the axis.

We use gradual changing color as the texture of flower's all components except the calyx and petal (see Fig.1(c)). Only one color is used for the calyx (see Fig.1(d)). And the petal has the real texture from the photo of the dissected plant, we assemble them together for integration and saving space.

3) Branches and Stems

Main points to both of them are their radius and holistic bending state. Then we apply the mesh smoothing technique to make them bend naturally.

The final shape of each organ with real texture are listed (see Fig.1). Then we use the L-system to control the organs' appearing positions and sizes over different developing stages.

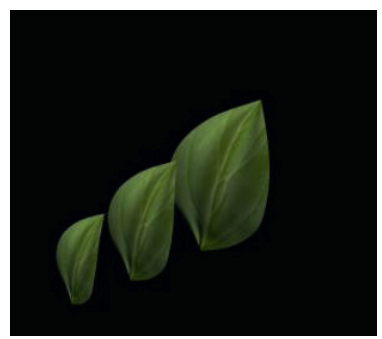

(a) leaf

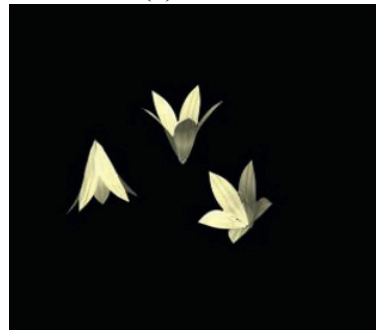

(c) chaplet composed by petals

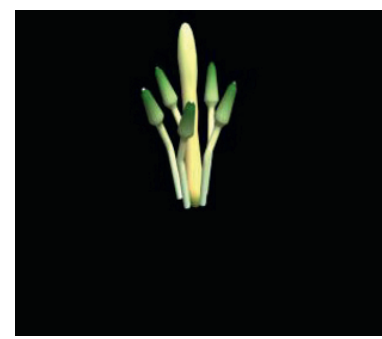

(b) stamen and pistil

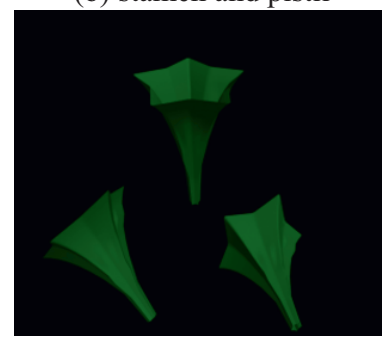

(d) calyx

Fig. 1. Some organs created by our method 


\subsection{Physiological approach}

We choose the formalism of L-systems as growing three-dimensional structures. L-systems emphasize plant topology, namely, the neighborhood relations between cells or larger plant modules (Prusinkiewicz et al.,1990).

The central concept of L-systems is rewriting. In general, rewriting is a technique for defining complex by successively replacing parts of simple initial objects with a set of rewriting rules or productions. It consists of triple table $\mathrm{G}=<\mathrm{V}, \mathrm{w}, \mathrm{P}>, \mathrm{V}$ is the alphabet, and $\mathrm{w}$ is the initiator, $\mathrm{P}$ is the productions list. One begins with two components: an initiator and a generator. The iteration process is: The $\mathrm{w}$ is the first iterative string, then replacing the character appeared in the last string by finding in the $\mathrm{P}$, repeating the step until all words have been replaced. Fig.2 gives the results evolved by the L-systems.

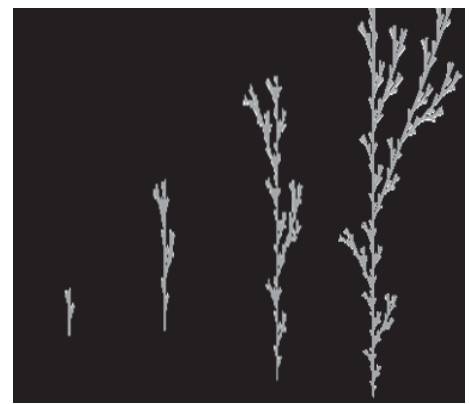

Fig. 2. The results that L-systems evolve

The evolving process is so similar to the development of the plant that we can use it to simulate the plant growing. For the L-systems can appoint the initiative state and productions list, we can apply these regulations to proceed the scheduled evolvement and form the complicate plant topology. Also we can appoint each of the letter in the alphabet the different graphical meaning (like the position and size et al.). And at different developing stages we analyze the string and give them appropriate graphical explanation.

\subsection{Integration of the visual effect and physiology}

This section illustrates the integration of the visual effect and L-systems. All the organs created in the section 2.1 have implemented the visual effect, then we import the organs into the L-systems. The whole structure of our improved L-system is given for supporting the integrality. We call it I-Lsystem which contains six modules: the save and fetch module, the grammar managing module, the organ transfer module, the alphabet appointing 
module, the evolving module and the rendering module. There are six steps to be proceeded, and Fig. 3 shows the structure of our I-L-system:

1) import the organs from the model library, and draw them by visual technology in real time, including displaying the texture correctly;

2) appoint every organ a special character that presents different graphical meaning in the L-grammar through the alphabet appointing module;

3) write the plant developing grammar of special growing physiology which will control the arrangement of the organs;

4) appoint the number of the iteration step, and the result of each step can be shown in the system evolving module;

5) set the camera position and light condition, and draw the growing scene through the rendering module;

6) save all the things finished in the former five steps, including saving the organ models, the plant developing grammar and the evolving result.

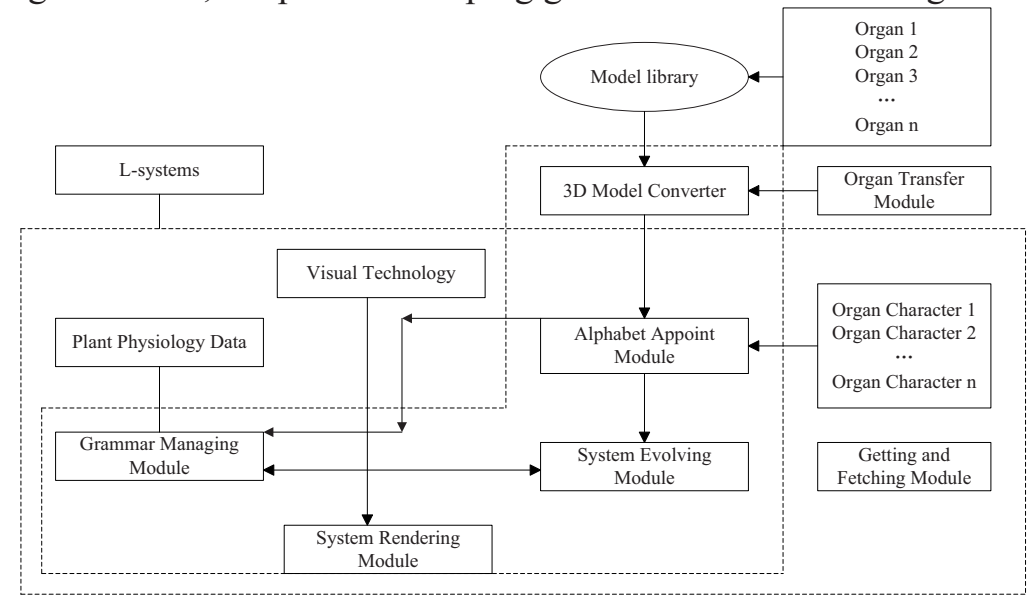

Fig. 3. The whole structure of the I-L-system

\section{MAIN PROCEDURE OF THE 3D MODEL CONVERTER}

We introduce the L-systems and analyze it in the second section, the findings show that L-systems are very good at exhibiting the plant topology due to its iteration process, and it also uses this way to produce the texture. To the realistic plant developing scene, there are various of texture of the organs, and these cost much iterative time. Compared to the real organ texture, the L-systems' are not so realistic for the former uses. Moreover when we want to remodel a new organ, the L-systems needs to recreate the organ, but our method support modifying the organs from the existing. 
This paper use $.3 \mathrm{ds}$ file as the input file format of the model library. Then we design a three dimension model converter to import these organs from the library into the L-systems.

\subsection{Data structure extracted from the .3ds file}

The $.3 \mathrm{ds}$ is the native file format of the Autodesk 3D Studio. And it has promulgated the standard file structure. The file is constructed by various of blocks. Each block has a head and body which can contain other blocks that can also contain other blocks. All of them are nested. Each head of the block consists of an ID and the block length, and the body contain the different data. The models determined by this format are the mesh style, that is, all of the organ surfaces are made up of triangles (others are quads and so on). Through analyzing, we find all the data can be extracted to following structure. Fig. 4 shows our extracted result for every organ, we will construct some data structure to store its data in our I-L-System.

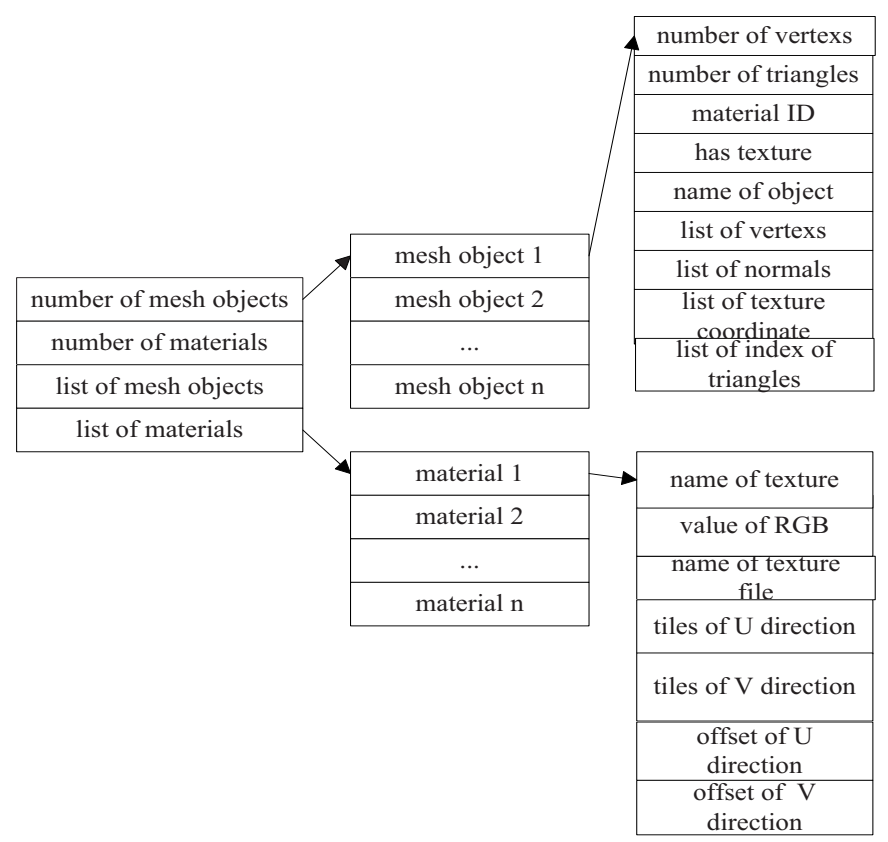

Fig. 4. The data structure extracted from the $.3 \mathrm{ds}$

\subsection{Two-part procedure of the 3D model converter}

In the last section, we have designed some data structure to store the organ data of the $.3 \mathrm{ds}$ model file. There are three important structures which are the model object, mesh object and material object. The relationship between 
them is every model object may contain many mesh objects, many kinds of texture, and every mesh object has the vertex, surface and material data, every material object has different texture. After confirming their existing format, we can design a three dimension model converter to transfer the model data to the format recognized by the L-systems.

The converter has two parts. The first is to read the data of the block in the $.3 \mathrm{~d}$ by iterative method to the appropriate data structure of the system, and the blocks we need to deal with are the main editing block, the material information block, the mesh object block and their interior subordinate block. Then do some relative computations including converting the coordinate of the model from the world space to the system space and computing the normal direction of each vertex of each mesh face. This can be done by dividing the number of the triangles sharing the vertex, and use it as the vertex's normal direction.

The second part is to make the imported model data correspond with the L-systems, that is, to let every organ has relevant character in the alphabet which may has the graphical meaning and let them be assembled by the developing grammar written according to the plant physiology. The process is to appoint a character to each model once we import it, when we start to draw the special developing scene, we read the string produced by the L grammar and analyse the character, if it is the organ character we call the draw module to display it. The two-part procedure is shown in Fig.5.

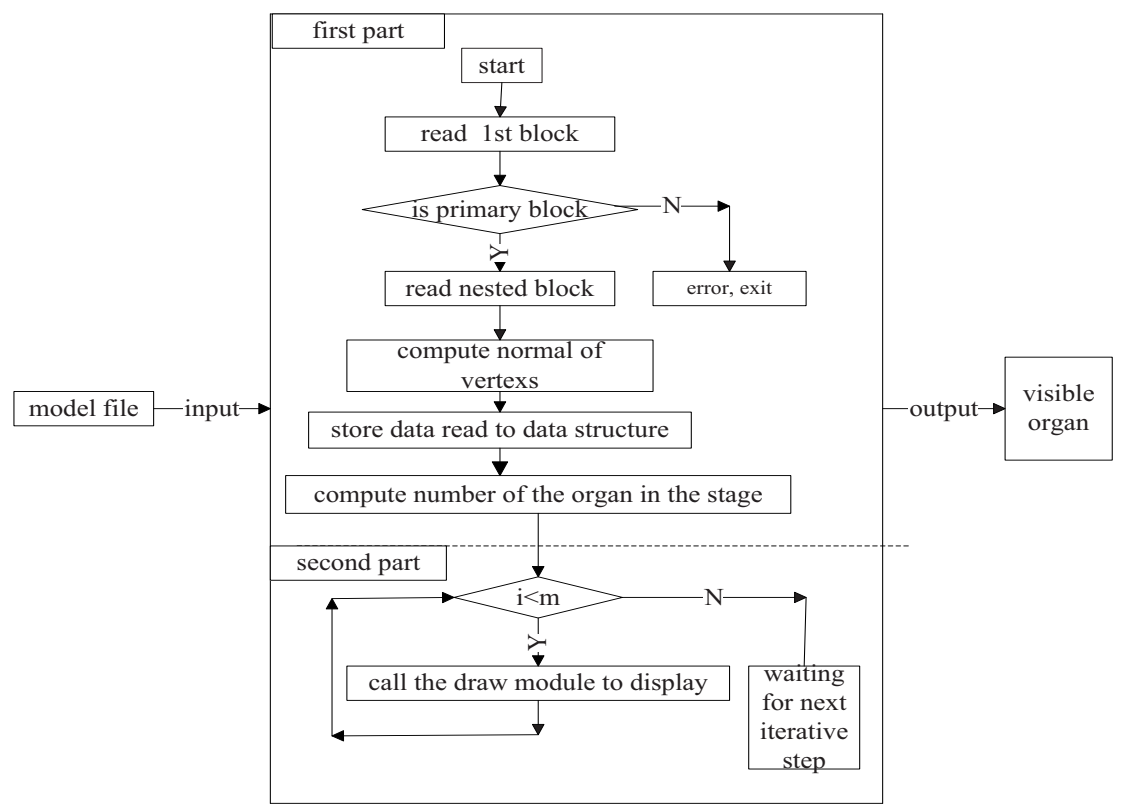

Fig. 5. The two-part procedure of the 3D model converter 


\section{EXPERIMENT AND ANALYSIS}

We use the organ models created by our method mentioned in the first section (see Fig.1), and import them into the I-L-System we propose. There is comparison of the growing scene created by the former L-systems and our I-L-System while they use the same developing grammar. Fig.6 gives the result of our system and the former L-Systems.

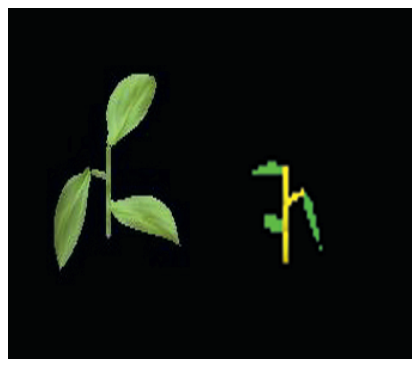

(a) the sixth step

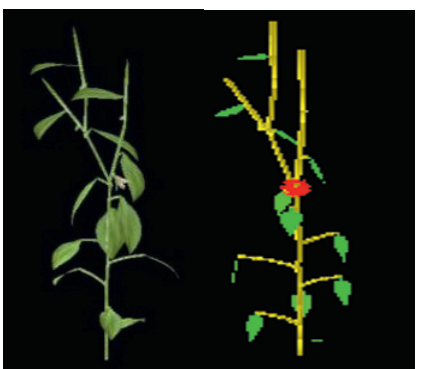

(c) the seventeenth step

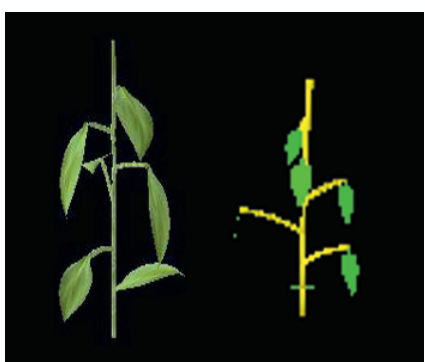

(b) the thirteenth step

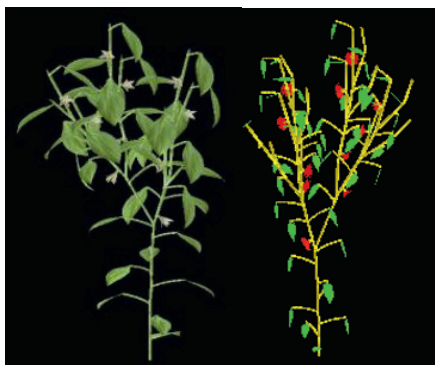

(d) the twenty-sixth step

Fig. 6. Four stages of developing scene created by I-L-System and former L-systems

Fig.6 clearly shows our method excels the former L-systems in the visual effect. Because our organs' texture are collected from the real leaves and petals which are mapped to the surface according to the shape of the organs, which helps to create more realistic scene. While L-systems only use the Bezier surface to create the organ models and use the simple color as the organs' texture.

We also give the time cost of the four developing stages of Fig.6 (see Table1).

Table1 indicates that we can render our developing scene in real time. To a developing stage that has $\mathrm{M}$ characters of the organs, and the average number of triangles of each mesh object is $\mathrm{N}$, the time cost must be $\mathrm{O}\left(\mathrm{M}^{*} \mathrm{~N}\right)$. It is a polynomial and when the iterative steps is not so many, the efficiency of our I-L-System is comparably high. 
Table1. Time cost of the four developing stages of our I-L-System

\begin{tabular}{ccc}
\hline Developing Stage & Iterative Steps & Cost(ms) \\
\hline \multirow{3}{*}{ A } & 6 & 186 \\
& 8 & 232 \\
& 10 & 295 \\
B & 12 & 373 \\
\cline { 2 - 3 } & 13 & 435 \\
& 15 & 560 \\
\cline { 2 - 3 } C & 17 & 778 \\
& 19 & 1107 \\
& 22 & 1944 \\
D & 24 & 2688 \\
\hline
\end{tabular}

\section{CONCLUSION}

The method based on the geometrical parameters leads to abstract and bad controllable modeling process, it also causes lacking of individual characteristics of the various plants' organs. The modeling method based on the image also has limitation that it has great dependence on the view point and needs plenty of photos taken from the plants. And the way based on the L-systems to create the organs can only support the Bezier surface to model them which have no realistic texture.

What we propose in this paper can solve the above problems. The results indicate that we can create the organs through outside modeling software which have a good revisability at any spatial position of the organ, it also helps us to enable them have abundant details on their surface. After finishing the models, we use the 3D model converter to import them and use the L-systems to assemble the whole organs created outside and call the rendering module to implement the visualization of the plant on computer in real time.

The environment is the living space for the plants, it has so big effect on the plants. While our I-L-System does not provide an interface for the environment to work on the plant. And the texture mapping method we use can only stick the smooth texture to the organ surface, however there must be rough on real organs, so we will concentrate on the two problems in future. 


\section{ACKNOWLEDGEMENTS}

Funding for this research was provided by the National High Technology Research and Development Program of China (863 Program) (2006AA10Z233) and the Ph.D. Programs Foundation of Ministry of Education of China (20050611027). The second author is grateful to the Computer College of Chongqing University for providing her with pursuing a Master degree at the Chongqing University. The third author is also grateful to the Computer College of Chongqing University for providing him with pursuing a Doctor degree at the Chongqing University.

\section{REFERENCES}

A.Lindenmayer, Mathmatical models for cellular interaction in development: parts I and II, Theor boil, 1968,18:280-315

E.A.Bier, K.R.Sloan, Two-part texture mapping, IEEE computer graphics and applications, 1986, 6(9):20-53

J.Weber, J.Penn, Creation and rendering of realistic trees, Computer graphics proceedings, Annual conference series, 1995:119-128

L.Mundermann, Y.Erasmus, B.Lane, et al., Quantitative modeling of arabidopisis development, Plant physiology, 2005, 139(2):960-968

M.Aono, T.Kunii, Botanical tree image generation, IEEE computer graphics and applications, 1984, 4(5):10-34

M.Jaeger, P.De Reffye, Basic comcepts of computer simulation of plant growth, Journal of biosciences, 1992, 17(3):275-291

Ma Xinming, Yang Juan, Xiong Shuping, et al. Reality and prospect of the virtual plant, Research of crop, 2003, 17(3):48-151(in Chinese)

P.De Reffye, C.Edelin, F.Jetal, Plant models faithful to botanical structure and development, Computer graphics, 1988, 22(4):151-158

P.De Reffye, J.Leroux, Study on plant growth behaviors simulated by the function-structural plant moedel-Greelab, Plant growth modeling and applications proceedings, 2003:118-128

P.Prusinkiewicz, A.Lindenmayer, The algorithmic beauty of plants, Springer-verlag, 1990

P.Prusinkiewicz, J.Hannan, Lindenmayer systems, Fractals and plants, 1989

R.Karwowski, P.Prosinkiewicz, Design and implenmentation of the $\mathrm{L}+\mathrm{C}$ modeling language, Electronic notes in theoretical computer science, 2003, 86:1-19

W.T.Reeves, Approximate and probabilistic algorithms for shading and rendering structured particle systems, Siggraph, 1985, 19(3):313-322 